\title{
Image analysis of small animal feeding behavior: An update
}

\author{
MARC ROWLEY \\ Berea College, Berea, Kentucky \\ AND \\ Frank Hanson \\ University of Maryland, Baltimore, Maryland
}

\begin{abstract}
Recently we published a design for an automated feeding behavior system (Rowley, Stitt, \& Hanson, 2003). One disadvantage of this system is that it relied on expensive, custom hardware. Here we present a modified version that replaces the custom hardware with a simple, commercially available webcam and modified the MATLAB analysis software as well. The new system is much less expensive and easier to implement, while still providing for bias-free collection of consumption data in a feeding behavior trial.
\end{abstract}

Our laboratory works with small animal feeding behavior. At the core of many of our experiments is a two-choice feeding assay that has taken many forms over the years. The assay itself is quite simple; an animal is placed into an arena containing six equidistant food items (typically plant leaf disks or wetted filter paper disks) and observed over the course of several hours. The animal's consumption of each food item is measured to determine feeding preference and rate.

Recently we published methods for an automated version of this test that used an array of CCD cameras to observe the arenas and a computer to collect images at desired timepoints (Rowley, Stitt, \& Hanson, 2003). These images are then processed by custom MATLAB software for bias-free monitoring of food item consumption. While this system has worked well for us, it relies on some custom hardware that is relatively expensive and difficult to reproduce. To simplify the equipment needed, and make it more accessible to other researchers, we have devised a new system that replaces this custom hardware with a single camera and improved software.

Commercial "webcam" cameras, coupled with an inexpensive software package, are an excellent solution for the simplification of such behavioral observations. A single highresolution webcam can be positioned to observe multiple behavioral arenas at once. They are easy to set up, inexpensive, and readily available for any computing platform. Many inexpensive software packages also exist to permit timed image collection from such cameras. Our new automated feeding assay system consists of a single webcam camera (iSight, Apple Computers) mounted on a ring-stand and positioned above a set of behavioral arenas. This camera is connected via FireWire to a Macintosh G4 computer running Oculus software (International Web, www.intlweb.com).
Our experiments with this new system follow the same simple procedures as those using the old hardware. An initial (time-zero) image is taken prior to introduction of the animals, and subsequent images are obtained at designated intervals for the duration of the experiment to record the disappearance of food. These images are stored on the hard disk until the user wishes to analyze them.

Image analysis is performed using a modified version of the MATLAB software presented in our previous publication (Rowley et al., 2003) in which each image contained data from only one behavioral arena. What differs is that now, each image contains the data for all of the arenas. The interactive software de-multiplexes this image resulting in a subset of arena-specific images. This is accomplished by having the user specify the number of arenas present and then clicking on an image to identify the location of each arena.

Flexibility of experimental conditions has also been increased by permitting variable numbers of food items, food item size, shape, location, and color. The user interacts with the program in defining these parameters by clicking within the first (time-zero) image, thus allowing for different sizes of food items, different camera distances from the arenas, or different camera resolutions.

Subsequent processing is as published in Rowley at al. (2003). Briefly, the images are masked to areas around the food items (Figure 1). These images are then converted to binary form and consumption of each food item over time is calculated by comparing the pixels of the food item at each time-point to the original pixel count from the timezero image. The program outputs both a table and a graph of consumption over time (Figure 2), and saves a data file for later analysis in MATLAB.

M. Rowley, marc_rowley@berea.edu 


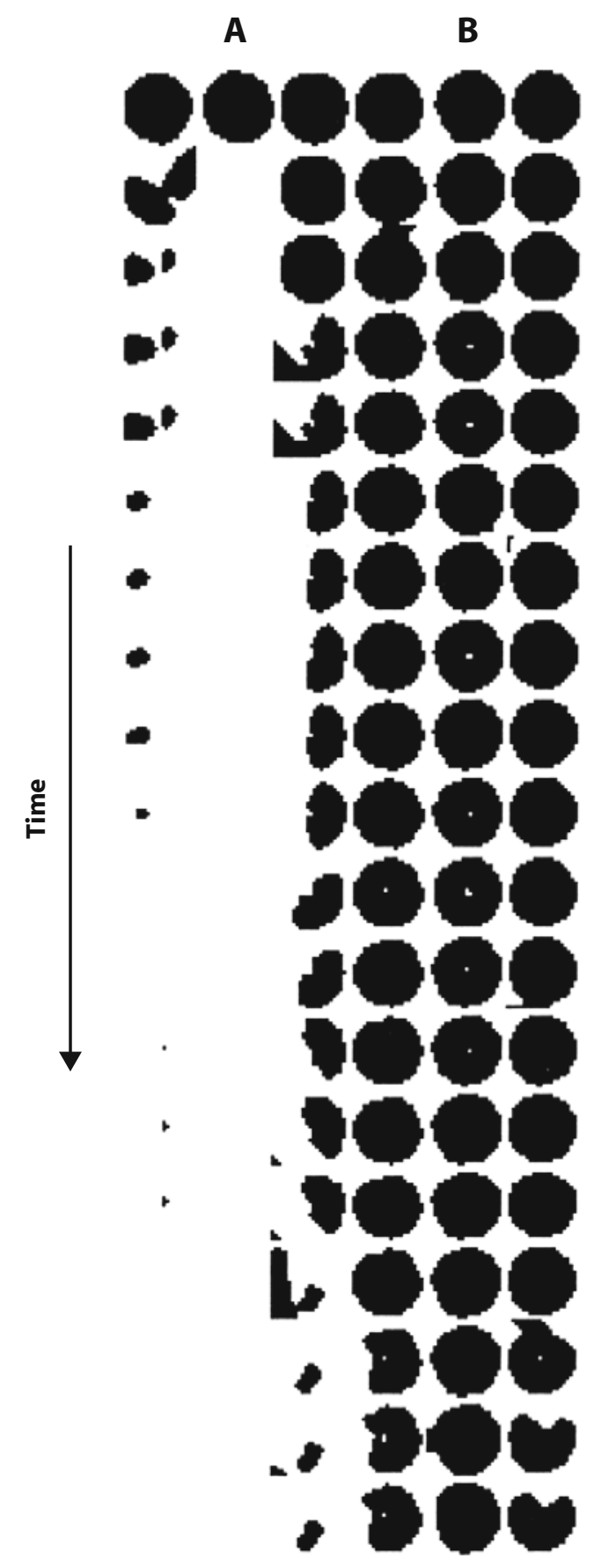

Figure 1. Sample data. The image shown above is a set of images obtained through our recording system, prior to final masking. Each circle is a food item, each row is a time-point (row 1 is the first, time-zero, image, row 2 is the second time-point and so on). Columns 1-3, food item A, are filter paper dises whetted with a control solution. Columns 4-6, food $B$, are filter paper dises whetted with a deterrent solution. Part of the caterpillar sometimes enters the image, as seen in rows $2-4$ and 16-17, and is masked out in later processing.

Throughout the process, user interaction is sometimes used in place of full automation. Doing so provides two important advantages. The first is that it keeps the software simple. Avoiding complex computer vision algorithms

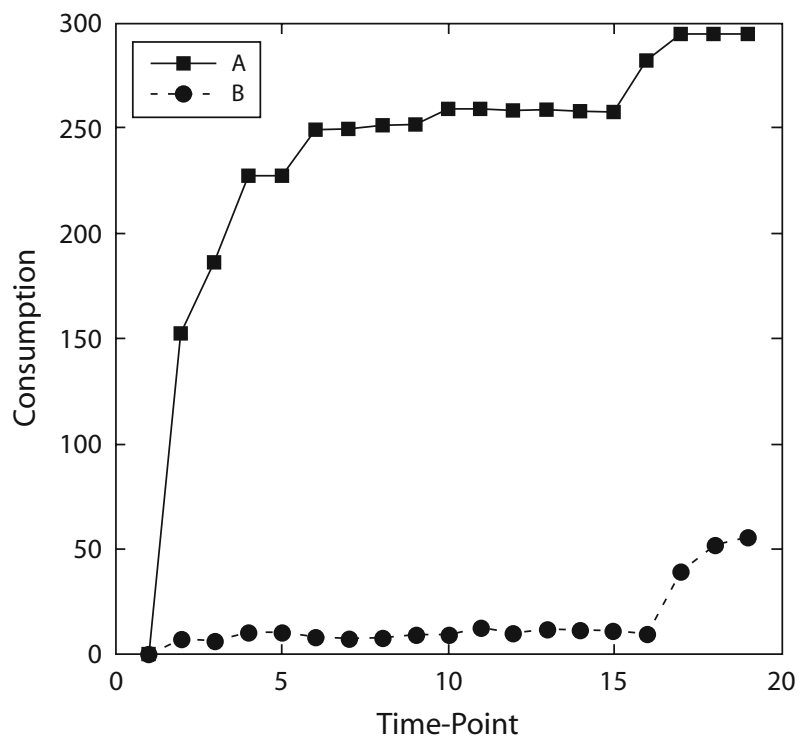

Figure 2. Sample output. This graph was produced after evaluating the images from Figure 1. The A group represents the first three food items (from left to right) in Figure 1, the B group is the last three food items. $y$-axis: Each food item consists of 100 consumption points $(100 \%)$, thus each group has a total possible consumption of 300. $x$-axis: Each image (row in Figure 1) is a timepoint, taken every 15 minutes (points 1-5) or 60 minutes (points 6-19). In this experiment, the A group items were preferred.

makes the software easily modifiable by anyone with a little programming knowledge. This will permit other researchers to customize it to their needs. The second advantage is that it allows the user to oversee the process while interacting with the images. Many problems, such as poor lighting in the arenas, can be quickly identified through this minor level of interaction.

In summary, while this new system requires a bit more user interaction, it ensures maximum flexibility in food and arena shape, with greatly reduced cost, time of construction, and maintenance. By increasing the complexity of the custom software only slightly, the need for custom hardware is eliminated permitting the use of off-the-shelf components (a computer, a webcam, and inexpensive webcam software).

Availability. A copy of this software is freely available through the MATLAB Central File Exchange (www.math works.com/matlabcentral) and is entitled "Feeding Monitor." In order to run this software, the user will need at least the base install of MATLAB (the student version is fine) and the Image Analysis Toolkit for MATLAB.

\section{AUTHOR NOTE}

Correspondence concerning this article should be addressed to M. Rowley, Department of Biology, Berea College, CPO 1972, Berea, KY 40404 (marc_rowley@berea.edu).

\section{REFERENCE}

Rowley, M., StitT, J. P., \& Hanson, F. E. (2003). Image analysis of small animal feeding behavior. Behavior Research Methods, Instruments, \& Computers, 35, 447-451.

(Manuscript received March 29, 2006; revision accepted for publication June 6, 2006.) 